\title{
Hot spot formation in electron-doped PCCO nanobridges
}

\author{
S. Charpentier, ${ }^{1}$ R. Arpaia, ${ }^{1}$ J. Gaudet, ${ }^{2}$ D. Matte, ${ }^{2}$ R. Baghdadi, ${ }^{1}$ T. Löfwander, ${ }^{3}$ D. Golubev,${ }^{4,5}$ P. Fournier, ${ }^{2}$ \\ T. Bauch, ${ }^{1}$ and F. Lombardi ${ }^{1, *}$ \\ ${ }^{1}$ Quantum Device Physics Laboratory, Department of Microtechnology and Nanoscience, Chalmers University of Technology, 41296 \\ Göteborg, Sweden \\ ${ }^{2}$ Canadian Institute for Advanced Research, Département de Physique, Université de Sherbrooke, J1K $2 R 1$ Sherbrooke, Canada \\ ${ }^{3}$ Applied Quantum Physics Laboratory, Department of Microtechnology and Nanoscience, Chalmers University of Technology, 41296 \\ Göteborg, Sweden \\ ${ }^{4}$ Low Temperature Laboratory (OVLL), Aalto University School of Science, P.O. Box 13500, FI-00076 Aalto, Finland \\ ${ }^{5}$ Institute of Nanotechnology, Karlsruhe Institute of Technology, D-76021 Karlsruhe, Germany
}

(Received 2 May 2016; published 1 August 2016)

\begin{abstract}
We have investigated the transport properties of optimally doped $\operatorname{Pr}_{2-x} \mathrm{Ce}_{x} \mathrm{CuO}_{4-\delta}$ (PCCO) nanobridges with width down to $100 \mathrm{~nm}$. The critical current density of the nanobridges approaches the Ginzburg-Landau theoretical limit, which demonstrates nanostructures with properties close to the as-grown films. The current voltage characteristics are hysteretic with a sharp voltage switch, of the order of a few millivolts, that we interpret with the occurrence of a hot spot formation. The values of the retrapping current and the voltage switch obtained by modeling the heat transport in the nanobridges are very close to the experimental ones. This feature, together with the extremely short recombination times, make PCCO nanostructures attractive candidates for ultrafast single photon detectors.
\end{abstract}

DOI: 10.1103/PhysRevB.94.060503

The microscopic mechanism at the origin of high critical temperature superconductors (HTS) is still elusive, due to the complexity of the systems, with various intertwined degrees of freedom and nanoscale local orders simultaneously at play [1]. Recent advances in nanopatterning techniques have paved the way for studying fundamental aspects of $\mathrm{HTS}$ on the nanoscale. $\mathrm{YBa}_{2} \mathrm{Cu}_{3} \mathrm{O}_{7-\delta}$ (YBCO) nanodots, for example, have shown the potentiality to uncover a small subdominant imaginary order parameter [2]. YBCO nanowires $[3,4]$ are another example of nanodevices that can open new experimental routes. Nanowires with dimensions comparable with the coherence length of the recently discovered charge density wave (CDW) [5,6] ordering (of the order of $10 \mathrm{~nm}[7,8])$, may allow one to study the interplay with the $d$-wave superconductivity and the possible relationship to the pseudogap state [9]. HTS nanoscale superconductivity can therefore be instrumental in clarifying the phenomena at the origin of the very complex phase diagram of cuprate superconductors.

Up to now, most of the attention has been devoted to the nanostructuring of the hole-doped cuprates, in particular YBCO [3,4,9-14]. The study of compounds from the electrondoped side of the cuprates phase diagram, for example, $\mathrm{Pr}_{2-x} \mathrm{Ce}_{x} \mathrm{CuO}_{4-\delta}$ (PCCO), has been hindered by the challenging synthesis; to obtain superconducting samples, one needs to simultaneously control the chemical doping and the oxygen content [15]. The experimental data available for the electron-doped cuprates suggest that there are both commonalities and differences between the two families [16]. Some features like the $d$-wave symmetry [17] of the order parameter have been shown to be universal suggesting the theoretical approaches to explain the pairing mechanism for hole-doped superconductors have to contemplate the

*floriana.lombardi@chalmers.se phenomenology of the electron-doped counterpart to the same extent.

In addition, HTS nanoscale devices can be of high relevance for applications where the short quasiparticles recombination times of $1-10$ ps typical of the cuprates [18,19] can be instrumental in achieving the ultimate limit of ultrafast single photon detection [20].

In this Rapid Communication we report a study of the transport properties of electron-doped PCCO nanobridges with dimensions down to $100 \mathrm{~nm}$ and carrying a supercurrent close to the theoretical Ginzburg-Landau value, a limit which is difficult to achieve even for conventional superconductors [21]. This is indicative of nanobridges which do not undergo degradation upon nanopatterning, preserving properties close to the as-grown film. The current-voltage characteristic of the nanobridges presents a sharp voltage switch, of the order of a few millivolts, from the superconducting state to the resistive state, that we interpret in terms of hot spot formation [22]. This feature, not found in state-of-the-art YBCO nanobridges $[23,24]$, is due to the different thermal conductivity between the two cuprate families and the different thermal boundary resistance between the films and the substrate, making the electron-doped compounds extremely attractive materials to engineer nanowire-based single photon detectors.

The 75-nm-thick $\operatorname{Pr}_{2-x} \mathrm{Ce}_{x} \mathrm{CuO}_{4-\delta}(x=0.15$ giving highest $T_{C}$ ) films were grown by pulsed laser ablation deposition on a (100) $\left(\mathrm{LaAlO}_{3}\right)_{0.3}-\left(\mathrm{Sr}_{2} \mathrm{TaAlO}_{6}\right)_{0.7}$ substrate, as described elsewhere [25]. A 35-nm-thick Au film was deposited in situ to ensure good adhesion to the PCCO. To define the nanostructures, we used electron-beam (e-beam) lithography in combination with a carbon mask and a gentle ion milling. More details on the patterning procedure are described elsewhere [4,23,26]. Figure 1(a) shows a scanning electron microscope (SEM) image of a typical nanowire. The rounded corners of the nanowire have been designed to minimize the effects of current crowding [4,27]. Figure 1(b) shows a 

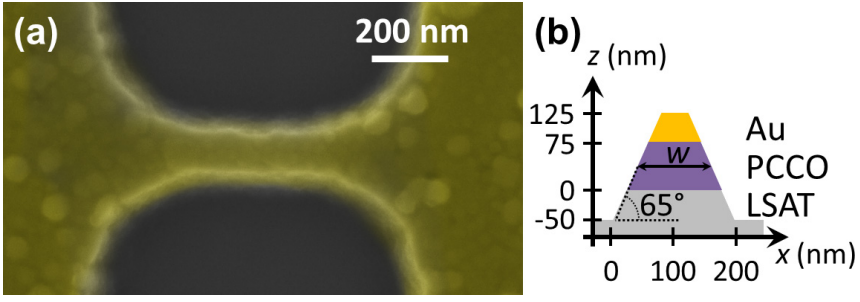

FIG. 1. (a) Scanning electron microscope picture of a 250-nmlong and 150-nm-wide nanowire. (b) Schematic view of the cross section of the nanowire. The slope of the bridge sidewalls is $65^{\circ}$, making the lateral extension of the $\mathrm{PCCO} / \mathrm{Au}$ bridge sidewalls roughly $58 \mathrm{~nm}$.

cross-section schematics of the nanowire. The slopes of the sidewalls of the nanowire have been inferred from SEM and atomic force microscopy (AFM).

Figure 2 shows the temperature dependence of the resistive transition for one of the measured nanowires capped with Au. The curve clearly presents two transition temperatures: the highest is the superconducting transition of the wide electrodes, and the lowest is attributed to the nanowire.

Current-voltage characteristics (IVCs) were measured at different temperatures, as shown in Fig. 3, in a four-point configuration. The critical current $I_{C}$ of the nanowire is determined with a voltage criterion of $10 \mu \mathrm{V}$. The value of $I_{C}$ allows one to determine the critical current density $J_{C}=I_{C} / A$, where $A=w \times h$ is the cross section of the nanowire, determined by SEM/AFM images [see Fig. 1(b)]. A common feature of the IVC, for all the measured nanowires, is the presence of a hysteresis whose appearance/disappearance in temperature is nanowire dependent. Also, the extrapolation of the IVC at high voltage does not intersect at the origin (as shown in the inset of Fig. 3), but at a finite excess current $I_{\text {exc }}$ due to Andreev reflection [28].

The quality and homogeneity of the superconducting nanowires can be assessed by comparing the critical current

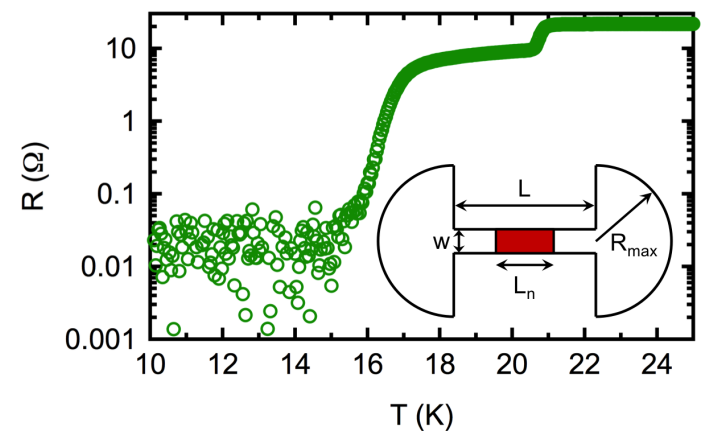

FIG. 2. Temperature dependence of the resistance of nanowire $\mathrm{S} 3$, measured with a dc current of $1 \mu \mathrm{A}$. Cooling down the nanowire, first the electrodes turn superconducting, then the nanowire. Inset: Schematic view of the nanowire used for the model. The nanowire has a length $L$, a width $w$, and a thickness $h$. In the model, we consider the formation of a hot spot that turns the central red part normal, over a length $L_{n}$. The temperature changes continuously from this normal part to the outer limits of the semicircle, where it equals the bath temperature.

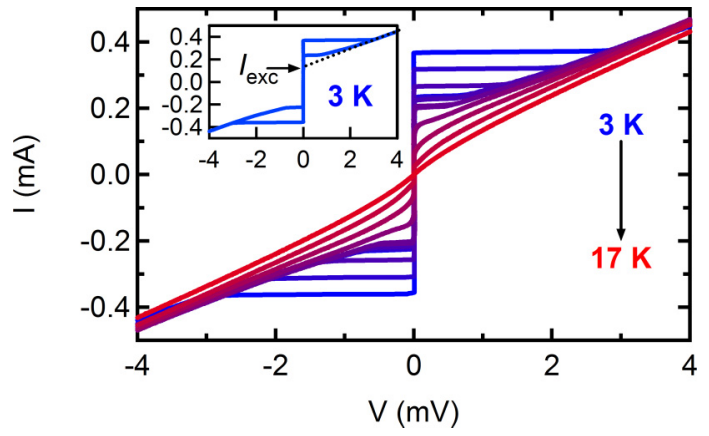

FIG. 3. IVC at different temperatures for nanowire S3. For clarity, we chose to show only one curve every $2 \mathrm{~K}$. Inset: IVC at low temperature, showing the switch between the superconducting state to the resistive state and the hysteresis and the presence of finite excess current $I_{\text {exc }}$.

density, obtained experimentally, with the theoretically expected value. In the case of nanowires with cross sections smaller than the Pearl length defined as $\lambda_{p}=\lambda_{0}^{2} / h$, where $\lambda_{0}$ is the London penetration depth, this value is given by the Ginzburg-Landau (GL) depairing limit:

$$
J_{\mathrm{GL}}=\frac{\Phi_{0}}{3^{3 / 2} \pi \mu_{0} \lambda_{0}^{2} \xi_{a b}},
$$

with $\Phi_{0}=2.15 \times 10^{-15} \mathrm{~T} \mathrm{~m}^{2}$ the superconducting flux quantum, $\mu_{0}$ the vacuum permeability, and $\xi_{a b}$ the coherence length in the $a b$ planes.

We can obtain the value of $\lambda_{0}$ by measuring the temperature dependence of the amplitude of the modulation of a nanowire-based superconducting quantum interference device. We fabricated nanoSQUIDs in the Dayem bridge configuration, as shown in Fig. 4(a). The critical current of a nanoSQUID, as a function of an externally applied magnetic field, is shown in Fig. 4(b). From this dependence we extract the critical current modulation depth $\Delta I_{C}(T)=I_{C}^{\max }(T)-$ $I_{C}^{\min }(T)$, which allows one to calculate the screening factor of the SQUID $\beta_{L}^{\text {expt. }}(T) \approx I_{C}^{\max }(T) / \Delta I_{C}(T)$ [29], assuming the Likharev-Yakobson expression for the current phase relation of the nanowire [30-32]. Furthermore, the screening factor can be expressed as $I_{C}^{\max }(T) L_{\text {loop }}(T) / \Phi_{0}$. Here $L_{\text {loop }}$ represents the total loop inductance of the nanoSQUID in our case governed by the kinetic inductance $L_{k}(T)$ of the nanowires. To extract the value of the London penetration depth, following Ref. [29], we have numerically calculated $L_{\text {loop }}$ at each temperature by solving the Maxwell and London equations describing the Meissner state on our nanoSQUID geometry, and assuming that the temperature dependence is given by the two-fluid model for the London penetration depth $\lambda_{L}=\lambda_{0} \frac{1}{\sqrt{1-\left(T / T_{C}\right)^{2}}}$. The experimental values of $\beta_{L}^{\text {expt. }}(T)$ can be fitted with the numerical one by using the London penetration depth $\lambda_{0}$ as the only free parameter. As shown in Fig. 4(c), there is good agreement between the data and numerical calculations in the whole temperature range using $\lambda_{0}=540 \mathrm{~nm}$. This value is roughly a factor of 2 higher than the values previously reported on single crystals of PCCO [33-35]. A similar increase was also reported for YBCO [36] and attributed to a higher level 

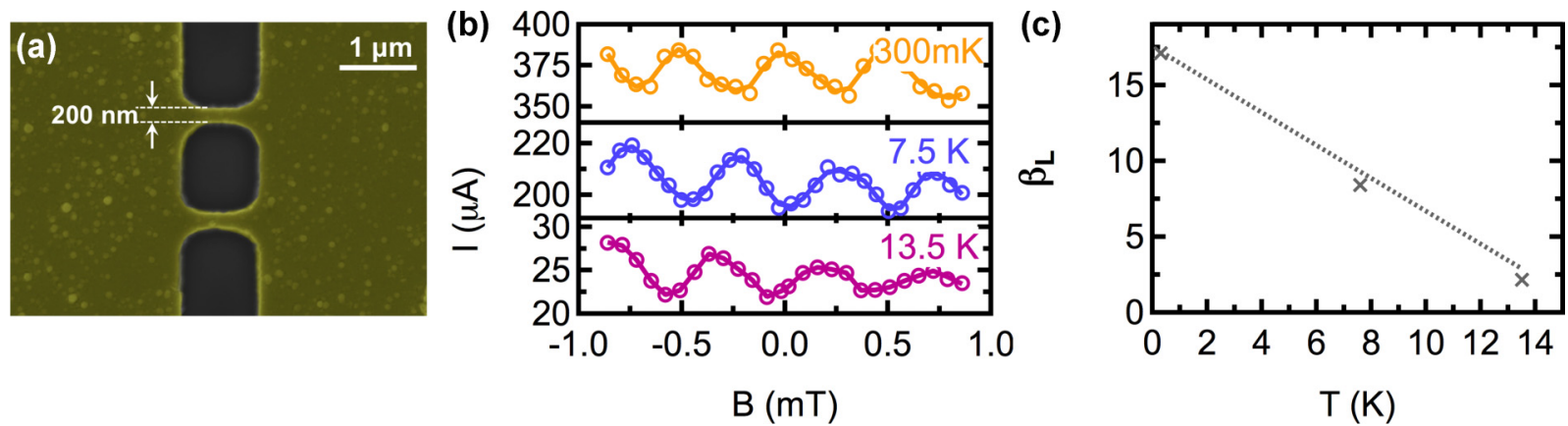

FIG. 4. (a) Scanning electron microscope picture of a nanoSQUID in the Dayem bridge configuration. The loop area is $1.2 \times 1.1 \mu \mathrm{m}^{2}$. (b) Critical current as a function of the applied magnetic field measured on the same device at $T=7.5 \mathrm{~K}$ (upper panel) and close to the critical temperature (lower panel). (c) Comparison between the experimental (crosses) and the theoretical (dashed line) values of the screening inductance factor $\beta_{L}$ as a function of the temperature. The experimental values of $\beta_{L}$ have been extracted from the critical current modulation depths, while the theoretical ones have been obtained by determining the loop inductance through numerical computation, assuming $\lambda_{L}=540 \mathrm{~nm}$.

of granularity in the thin films compared to single crystals. It has also been observed in YBCO nanowires [37].

We also measured the value of $B_{c 2}=\Phi_{0} / 2 \pi \xi_{a b}^{2}=5.1 \mathrm{~T}$, which gives us an estimate for $\xi_{a b} \simeq 8 \mathrm{~nm}$ at $2 \mathrm{~K}$, in agreement with previous measurements $[35,38,39]$. With the value of $\lambda_{0}$ and $\xi_{a b}$ we obtained a theoretical depairing critical current density $J_{C}^{\max }=4.3 \times 10^{6} \mathrm{~A} / \mathrm{cm}^{2}$ at $T=2 \mathrm{~K}$. The $J_{C}$ values of our 100- and 200-nm-long nanowires with width $w$ down to $100 \mathrm{~nm}$ are in the range $2.1-4.2 \times 10^{6} \mathrm{~A} / \mathrm{cm}^{2}$, therefore approaching the theoretical limit and confirming the high quality of the devices. This fact is a strong indication that the switching observed in the IVC is not caused by the presence of local grain boundaries and/or defects. The length of our bridges, $L \simeq 200 \mathrm{~nm}$, is much larger than the superconducting coherence length $\left(\xi_{a b} \simeq 8 \mathrm{~nm}\right)$. In this limit such nanobridges cannot be considered Josephson junctions [32]. Therefore, we can rule out any Josephson dynamics related model such as the resistively and capacitively shunted junction model [40,41] being responsible for the observed hysteresis.

Instead, the origin of thermal hysteresis can be understood as follows. In the superconducting state, $I<I_{C}$, the temperature of the quasiparticles inside the wire is equal to that of the substrate $T_{0}$. As soon as the GL maximum critical current is reached, the voltage drop across the wire generates a hot spot caused by Joule heating. At sufficiently high bias, the whole wire becomes normal. If one now reduces the bias current from high values, the normal part gradually shrinks. Switching back to the superconducting state occurs at the retrapping current, $I_{r}$, which is lower than the critical one, $I_{r}<I_{C}\left(T_{0}\right)$, since the Joule heating in the hot spot elevates the temperature of the wire. $I_{r}$ is determined by the condition $I_{r}=I_{C}\left(T_{\mathrm{NS}}\right)$, where $T_{\mathrm{NS}}>T_{0}$ is the minimal temperature at the boundary between normal and superconducting parts (NS boundary) of the wire at which the normal hot spot in the wire still exists. This mechanism is well known for low temperature superconductors $[42,43]$.

To make our analysis more quantitative, we propose a simple model in the spirit of Refs. [43-45]. We consider the wire of length $L$, width $w$, and thickness $h$ as shown in the inset of Fig. 2. We assume that the central part of it with length $L_{n}$ becomes normal due to Joule heating, and the power $I V$ is generated in this segment. This power is carried away into the leads by quasiparticles, which are supposed to be decoupled from the phonons on a scale comparable with the wire length [46]. We also assume that both leads are identical and that the electronic temperature at the edges of the semicircular parts of the leads, shown in the inset of Fig. 2, equals to $T_{0}$. Finally, we assume that large thermal boundary resistance is present at the interface between the substrate and the film. In this case, applying the boundary condition at the NS interface we obtain the equation for temperature in the superconducting part of the wire in the form

$$
w h \kappa_{\mathrm{el}}(T) \frac{d T}{d x}=-\frac{I V}{2}, \quad \frac{L_{n}}{2}<x<\frac{L}{2},
$$

where $\kappa_{\mathrm{el}}(T)$ is the electronic part of the thermal conductibility. A similar equation determines the temperature profile in the leads. The solution of these equations is described in the Supplemental Material [47]. It has been experimentally shown [16] that $\kappa_{\mathrm{el}}(T)$ in PCCO is a linear function of temperature, $\kappa_{\mathrm{el}}(T)=\beta T\left(\beta \approx 0.06 \mathrm{~W} / \mathrm{K}^{2} \mathrm{~m}\right)$, in the low temperature limit $T \lesssim T^{*} \sim 0.5 \mathrm{~K}$. In this regime it probably exhibits a universal disorder independent behavior similarly to other $d$-wave cuprate superconductors $[48,49]$. In the normal state, i.e., at $T>T_{C}$, the Wiedemann-Franz law should hold and $\kappa_{\mathrm{el}}(T)=$ $\mathcal{L} T / \rho$, where $\mathcal{L}=\pi^{2} k_{B}^{2} / 3 e^{2} \approx 2.45 \times 10^{-8} \mathrm{~W} \Omega / \mathrm{K}^{2}$ is the

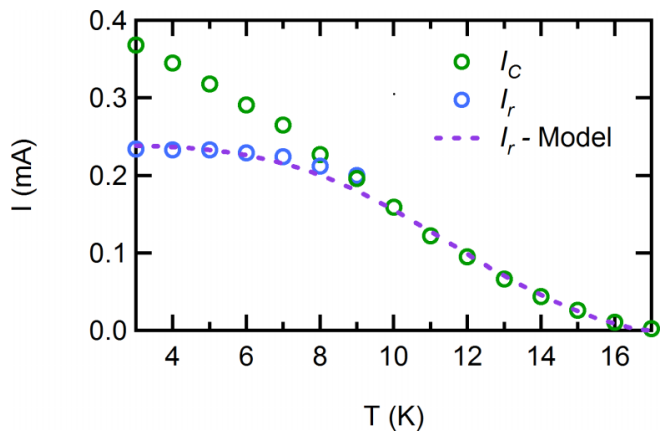

FIG. 5. Experimental critical and retrapping currents (circles) as a function of the temperature for nanowire S3. Calculated retrapping current, obtained from the numerical solution of Eq. (4) with $\alpha=3.7$. 
TABLE I. Parameters of five nanowires with different length $L$ and comparison of the values obtained for the retrapping current and voltage from the experimental curves (expt.) to the one calculated with the model (model), at $T=3 \mathrm{~K}$.

\begin{tabular}{lccccccccc}
\hline \hline Device & $L(\mathrm{~nm})$ & $R_{N}(\Omega)$ & $T_{C}^{\text {wire }}(\mathrm{K})$ & $J_{c}\left(10^{6} \mathrm{~A} / \mathrm{cm}^{2}\right)$ & $I_{\text {exc }}(\mathrm{mA})$ & $I_{r}^{\text {expt. }}$ & $I_{r}^{\text {model }}$ & $V_{r}^{\text {expt. }}$ & $V_{r}^{\text {model }}$ \\
\hline S1 & 200 & 17.42 & 17 & 2.1 & 0.122 & 0.148 & 0.151 & 0.44 \\
S2 & 100 & 12.35 & 17 & 2.2 & 0.127 & 0.165 & 0.169 & 0.37 \\
S3 & 200 & 12.26 & 17 & 3.8 & 0.117 & 0.233 & 0.238 & 0.7 \\
S4 & 100 & 11.82 & 18 & 4.2 & 0.125 & 0.275 & 0.261 & 1.02 \\
S5 & 200 & 9.09 & 16.5 & 3.8 & 0.141 & 0.305 & 0.302 & 0.84 \\
\hline \hline
\end{tabular}

Lorentz number and $\rho$ is the resistivity of the material in the normal state [47]. Unfortunately, the temperature dependence of the electronic thermal conductivity of PCCO is not precisely known in the intermediate temperature interval $T^{*}<T<T_{C}$, which is relevant for our measurements. Provided the impurity scattering of electrons is sufficiently weak, its competition with the phonon scattering may result in nonmonotonic $\kappa_{\mathrm{el}}(T)$ with a peak slightly below the critical temperature [50], as observed in YBCO. However, a reasonable assumption is that in our nanowires, $\kappa_{\mathrm{el}}(T)$ monotonically decreases with decreasing temperature in the temperature interval $2 \mathrm{~K}<T<8 \mathrm{~K}$, where the hysteretic IVC are observed (see Fig. 5). This assumption is supported by measurements of the thermal conductivity in bulk PLCCO, NCCO, and PCCO samples [51-53], in which a monotonous dependence has been observed, even though in these measurements it was not possible to separate the electronic contribution from the phonon one [46]. Following the predictions of Refs. [48,50], we use a simple phenomenological model $\kappa_{\mathrm{el}}(T)=\alpha \mathcal{L} T^{3} / \rho T_{C}^{2}$ for $T^{*}<T<8 \mathrm{~K}$. This leaves only $\alpha$ as an adjustable parameter in the model. [47]

Solving Eq. (2) and a similar equation for the leads we find

$$
\frac{I V}{2}=\frac{\alpha \mathcal{L}\left(T_{\mathrm{NS}}^{4}-T_{0}^{4}\right)}{4 T_{C}^{2}\left(R_{\mathrm{wire}}^{S}+R_{\text {lead }}\right)},
$$

where $R_{\text {wire }}^{S}=R_{N}\left(1-L_{n} / L\right) / 2$ is the normal state resistance of the wire segment $L_{n} / 2<x<L / 2, R_{N}$ is the normal state resistance of the whole wire, and $R_{\text {lead }}$ is the normal state resistance of the semicircle part of a lead shown in the inset of Fig. [2]. Equation (3) should be supplemented by the conditions $I=I_{C}\left(T_{\mathrm{NS}}\right)$ and $V=\left(I-I_{\mathrm{exc}}\right) R_{N} L_{n} / L$. From these equations we find both $L_{n}$ and $T_{\mathrm{NS}}$ as a function of the bias current. Under simplifying assumption $R_{\text {wire }}^{S} \gg R_{\text {lead }}$, which is approximately valid in our experiment, the retrapping occurs at $L_{n}=L / 2$ [47]. It leads to the expressions for the retrapping current,

$$
I_{r}=I_{C}\left(T_{\mathrm{NS}}\right), \frac{I_{r}\left(I_{r}-I_{\mathrm{exc}}\right) R_{N}}{4}=\frac{\alpha \mathcal{L}\left(T_{\mathrm{NS}}^{4}-T_{0}^{4}\right)}{T_{C}^{2} R_{N}},
$$

and the retrapping voltage, $V_{r}=\left(I_{r}-I_{\text {exc }}\right) R_{N} / 2$. The calculated retrapping current as a function of temperature is shown in Fig. 5. The best agreement to our experimental data is found with the adjustable parameter $\alpha=3.7$. This is a relatively big value: with this number, the low temperature formula for the thermal conductivity will exceed the normal state Wiedemann-Franz expression at $T=T_{C}$, which indicates the possible existence of a peak in the $\kappa_{\mathrm{el}}(T)$ dependence in agreement with the theory [50] and with experiments with other $d$-wave superconductors [51,54]. It is, however, unlikely that there is a significant peak in the electronic contribution to the thermal conductivity below $8 \mathrm{~K}$, since this would induce a discontinuity in the temperature dependence of the retrapping current and since it has not been seen in experiments [53]. Using $\alpha=3.7$, we calculated the values for the retrapping current and voltage for several nanowires, as presented in Table I. The obtained values of $I_{r}$ are in excellent agreement with the experimental ones, also reported in the Table I. However, for $V_{r}$, the experimental values may exceed the theoretical ones by up to $30 \%$. It is expected, since $V_{r}$ is more sensitive to the model assumptions about the value of $L_{n}$ at the retrapping. The latter may exceed $L / 2$, which our model predicts, if one takes into account the finite resistance of the leads.

In conclusion, we have reported nanopatterned devices made from an electron-doped cuprate. The quality of the nanowires is demonstrated by the high current density they can carry without dissipation and by the observation of the nanoSQUIDs magnetic field modulations over the whole temperature range up to $T_{C}$. The IVC of the devices shows a clear switch from the superconducting to the resistive state, in contrast to what is observed in YBCO nanowires. Our results can be explained in terms of thermal hysteresis, where a hot spot is created in the wire. In our model, we calculate how the heat is dissipated. The estimated values of the retrapping current are in excellent agreement with the measured ones. The presence of the switching voltage makes PCCO an attractive material for superconducting nanowire single photon detectors.

This work is supported by the Swedish Research Council (VR) and the Knut and Alice Wallenberg Foundation. We thank K. D. Truong, M. Dion, and S. Pelletier for technical assistance. We acknowledge the financial support of CIfAR, Canada Foundation for Innovation (CFI), NSERC (Canada) and FQRNT (Québec).
[1] M. Hashimoto, I. M. Vishik, R.-H. He, T. P. Devereaux, and Z.-X. Shen, Nat. Phys. 10, 483 (2014).
[2] D. Gustafsson, D. Golubev, M. Fogelström, T. Claeson, S. Kubatkin, T. Bauch, and F. Lombardi, Nat. Nanotechnol. 8, 25 (2013). 
[3] K. Xu and J. R. Heath, Nano Lett. 8, 3845 (2008).

[4] S. Nawaz, R. Arpaia, F. Lombardi, and T. Bauch, Phys. Rev. Lett. 110, 167004 (2013).

[5] P. Abbamonte, A. Rusydi, S. Smadici, G. D. Gu, G. A. Sawatzky, and D. L. Feng, Nat. Phys. 1, 155 (2005).

[6] M. Hücker, M. V. Zimmermann, G. D. Gu, Z. J. Xu, J. S. Wen, G. Xu, H. J. Kang, A. Zheludev, and J. M. Tranquada, Phys. Rev. B 83, 104506 (2011).

[7] G. Ghiringhelli, M. Le Tacon, M. Minola, S. Blanco-Canosa, C. Mazzoli, N. B. Brookes, G. M. De Luca, A. Frano, D. G. Hawthorn, F. He, T. Loew, M. Moretti Sala, D. C. Peets, M. Salluzzo, E. Schierle, R. Sutarto, G. A. Sawatzky, E. Weschke, B. Keimer, and L. Braicovich, Science 337, 821 (2012).

[8] J. Chang, E. Blackburn, A. T. Holmes, N. B. Christensen, J. Larsen, J. Mesot, R. Liang, D. A. Bonn, W. N. Hardy, A. Watenphul, M. V. Zimmermann, E. M. Forgan, and S. M. Hayden, Nat. Phys. 8, 871 (2012).

[9] J. A. Bonetti, D. S. Caplan, D. J. Van Harlingen, and M. B. Weissman, Phys. Rev. Lett. 93, 087002 (2004).

[10] N. Curtz, E. Koller, H. Zbinden, M. Decroux, L. Antognazza, O. Fischer, and N. Gisin, Supercond. Sci. Technol. 23, 045015 (2010).

[11] I. Sochnikov, A. Shaulov, Y. Yeshurun, G. Logvenov, and I. Bozovic, Nat. Nanotechnol. 5, 516 (2010).

[12] G. Papari, F. Carillo, D. Stornaiuolo, L. Longobardi, F. Beltram, and F. Tafuri, Supercond. Sci. Technol. 25, 035011 (2012).

[13] D. Levi, A. Shaulov, A. Frydman, G. Koren, B. Ya. Shapiro, and Y. Yeshurun, Europhys. Lett. 101, 67005 (2013).

[14] R. Arpaia, S. Charpentier, R. Toskovic, T. Bauch, and F. Lombardi, Physica C 506, 184 (2014).

[15] J. Gauthier, S. Gagné, J. Renaud, M. È. Gosselin, P. Fournier, and P. Richard, Phys. Rev. B 75, 024424 (2007).

[16] N. P. Armitage, P. Fournier, and R. L. Greene, Rev. Mod. Phys. 82, 2421 (2010).

[17] C. C. Tsuei and J. Kirtley, Rev. Mod. Phys. 72, 969 (2000).

[18] M. Lindgren, M. Currie, C. Williams, T. Y. Hsiang, P. M. Fauchet, R. Sobolewski, S. H. Moffat, R. A. Hughes, J. S. Preston, and F. A. Hegmann, Appl. Phys. Lett. 74, 853 (1999).

[19] Y. B. Long, L. Zhao, B. R. Zhao, X. G. Qiu, C. Y. Zhang, P. M. Fu, L. Wang, Z. G. Zhang, S. P. Zhao, Q. S. Yang, and G. P. Wang, Physica C 436, 59 (2006).

[20] G. N. Gol'tsman, O. Okunev, G. Chulkova, A. Lipatov, A. Semenov, K. Smirnov, B. Voronov, A. Dzardanov, C. Williams, and R. Sobolewski, Appl. Phys. Lett. 79, 705 (2001).

[21] K. Xu, P. Cao, and J. R. Heath, Nano Lett. 10, 4206 (2010).

[22] D. Golubev, F. Lombardi, and T. Bauch, Physica C 506, 174 (2014).

[23] R. Arpaia, S. Nawaz, F. Lombardi, and T. Bauch, IEEE Trans. Appl. Supercond. 23, 1101505 (2013).

[24] R. Arpaia, M. Ejrnaes, L. Parlato, F. Tafuri, R. Cristiano, D. Golubev, R. Sobolewski, T. Bauch, F. Lombardi, and G. Pepe, Physica C 509, 16 (2015).

[25] G. Roberge, S. Charpentier, S. Godin-Proulx, P. Rauwel, K. D. Truong, and P. Fournier, J. Cryst. Growth 311, 1340 (2009).

[26] S. Nawaz, R. Arpaia, T. Bauch, and F. Lombardi, Physica C 495, 33 (2013).

[27] J. R. Clem and K. K. Berggren, Phys. Rev. B 84, 174510 (2011).
[28] G. E. Blonder, M. Tinkham, and T. M. Klapwijk, Phys. Rev. B 25, 4515 (1982).

[29] R. Arpaia, M. Arzeo, S. Nawaz, S. Charpentier, F. Lombardi, and T. Bauch, Appl. Phys. Lett. 104, 072603 (2014).

[30] M. Y. Kupriyanov and K. K. Likharev, Fiz. Tverd. Tela 16, 2829 (1974).

[31] K. K. Likharev and L. A. Yakobson, Sov. Phys. Tech. Phys. 20, 950 (1976).

[32] K. Likharev, Rev. Mod. Phys. 51, 101 (1979).

[33] R. Prozorov, R. W. Giannetta, A. Carrington, P. Fournier, R. L. Greene, P. Guptasarma, D. G. Hinks, and A. R. Banks, Appl. Phys. Lett. 77, 4202 (2000).

[34] J. A. Skinta, Mun-Seog Kim, T. R. Lemberger, T. Greibe, and M. Naito, Phys. Rev. Lett. 88, 207005 (2002)

[35] C. C. Homes, R. P. S. M. Lobo, P. Fournier, A. Zimmers, and R. L. Greene, Phys. Rev. B 74, 214515 (2006).

[36] A. G. Zaitsev, R. Schneider, G. Linker, F. Ratzel, R. Smithey, P. Schweiss, J. Geerk, R. Schwab, and R. Heidinger, Rev. Sci. Instrum. 73, 335 (2002).

[37] R. Arpaia, D. Golubev, R. Baghdadi, M. Arzeo, G. Kunakova, S. Charpentier, S. Nawaz, F. Lombardi, and T. Bauch, Physica C 506, 165 (2014).

[38] M. M. Qazilbash, A. Koitzsch, B. S. Dennis, A. Gozar, H. Balci, C. A. Kendziora, R. L. Greene, and G. Blumberg, Phys. Rev. B 72, 214510 (2005)

[39] Y. Dagan, R. Beck, and R. L. Greene, Phys. Rev. Lett. 99, 147004 (2007).

[40] W. C. Stewart, Appl. Phys. Lett. 12, 277 (1968).

[41] D. E. McCumber, J. Appl. Phys. 39, 3113 (1968).

[42] A. V. Gurevich and R. G. Mints, Rev. Mod. Phys. 59, 941 (1987),

[43] M. Tinkham, J. U. Free, C. N. Lau, and N. Markovic, Phys. Rev. B 68, 134515 (2003).

[44] H. Courtois, M. Meschke, J. T. Peltonen, and J. P. Pekola, Phys, Rev. Lett. 101, 067002 (2008).

[45] N. Kumar, T. Fournier, H. Courtois, C. B. Winkelmann, and A. K. Gupta, Phys. Rev. Lett. 114, 157003 (2015).

[46] M. F. Smith, J. Paglione, M. B. Walker, and L. Taillefer, Phys. Rev. B 71, 014506 (2005)

[47] See Supplemental Material at http://link.aps.org/supplemental/ 10.1103/PhysRevB.94.060503 for details about the hot spot model used to describe the hysteresis in the IVC of the PCCO nanowires.

[48] Y. Sun and K. Maki, Europhys. Lett. 32, 355 (1995).

[49] M. J. Graf, S.-K. Yip, J. A. Sauls, and D. Rainer, Phys. Rev. B 53, 15147 (1996).

[50] T. Löfwander and M. Fogelström, Phys. Rev. Lett. 95, 107006 (2005).

[51] X. F. Sun, Y. Kurita, T. Suzuki, S. Komiya, and Y. Ando, Phys. Rev. Lett. 92, 047001 (2004).

[52] R. Larger, M.S. thesis, Université de Sherbrooke, 2005.

[53] X. Zhao, Z. Y. Zhao, B. Ni, J. C. Wu, F. B. Zhang, J. D. Song, S. J. Li, X. F. Sun, and X. G. Li, Phys. Rev. B 90, 024518 (2014).

[54] M. Sutherland, D. G. Hawthorn, R. W. Hill, F. Ronning, S. Wakimoto, H. Zhang, C. Proust, E. Boaknin, C. Lupien, L. Taillefer, R. Liang, D. A. Bonn, W. N. Hardy, R. Gagnon, N. E. Hussey, T. Kimura, M. Nohara, and H. Takagi, Phys. Rev. B 67, 174520 (2003). 